\title{
Misinformation and the Mindsponge mechanism of trust
}

\author{
Tam-Tri Le, Minh-Hoang Nguyen *, Quan-Hoang Vuong \\ Centre for Interdisciplinary Social Research, Phenikaa University, Yen Nghia Ward, Ha Dong \\ District, Hanoi 100803, Vietnam \\ * Correspondence: hoang.nguyenminh@phenikaa-uni.edu.vn
}

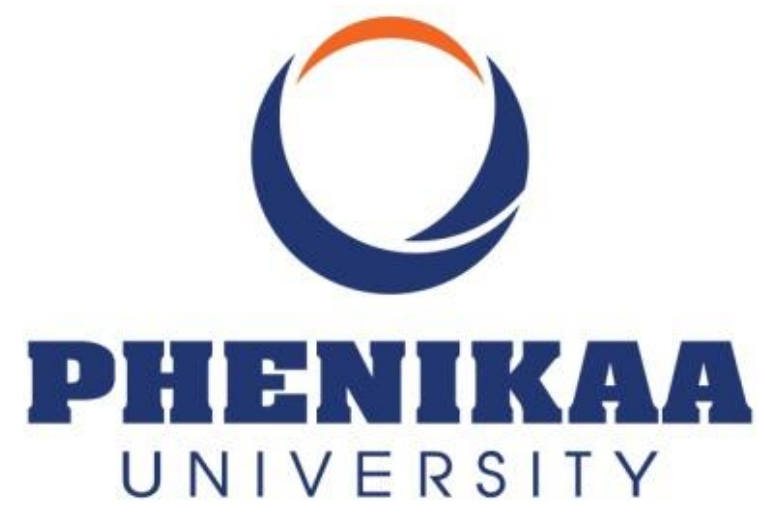

\begin{abstract}
Misinformation is a serious issue, especially during the COVID-19 global health crisis. In this digital era, people are expected to process a huge amount of information every day. Based on the Mindsponge framework information processing, we explore the role of trust as a facilitator within the information filtering process, a natural energy-saving mechanism of how the human mind works. This mechanism can help explain how modern humans are prone to misinformation.

$* * * * * * * *$
\end{abstract}

Since the beginning of the Covid-19 pandemic, governments and the public have been fighting against not only the viral disease but also the "infodemics" - the wide and rapid spread of false and misleading information. The dissemination of inaccurate information can lead to problematic consequences (e.g., confusion and doubt towards the trustworthiness of the information, subsequent reliance on misleading information) (Rapp \& Salovich, 2018) and prevent the timely and effective adoption of public health recommendations and measures among the public (Waszak, Kasprzycka-Waszak, \& Kubanek, 2018). Evidently, "scientific-sounding misinformation" is a strong predictor of vaccination hesitancy (Loomba, de Figueiredo, Piatek, de Graaf, \& Larson, 2021).

Besides the exposure to misinformation and the lack of accessibility to credible sources of information, public distrust is also a fundamental issue that drives infodemics. Among the 812,000 times that anti-vaccine content was shared or posted on Facebook and Twitter between 1 
February and 16 March 2021, 65\% was attributable to the Disinformation Dozen (Center for Countering Digital Hate, 2021). The Disinformation Dozen consists of highly influenced individuals that obtain millions of followers across social platforms. The burning of wireless towers in the United Kingdom due to the belief in a conspiracy theory linking the spread of coronavirus to 5G wireless technology (Satariano \& Alba, 2020) also solidifies the distrust's impacts on the infodemics' severity. Considering the vital role of trust in tackling misinformation, the current essay uses the Mindsponge mechanism supported by some evidence in biology to explore the function of trust as a mechanism for conserving energy during the information evaluation process. This mechanism, therefore, helps an individual meet their demand of information given the usable energy constraint.

The Mindsponge framework was proposed by Vuong and Napier (2015) and later expanded (Nguyen et al., 2021; Q.-H. Vuong, Nguyen, \& Le, 2021a) to explain how an individual absorbs new information and discards unwanted information. The framework has five major components: 1) mindset, 2) comfort zone, 3) multi-filtering system, 4) cultural and ideological setting, and 5) information. When information enters the mind, it is evaluated by the multi-filtering system based on references from the mindset. The product of the filtering process is the decision to whether accept the information into the mindset, discard it, or temporarily keep it in the comfort zone for later evaluation.

The filtering process includes subjective cost-benefit judgments and trust evaluation. Costbenefit judgments attach values to the information by connecting and comparing it with the references stored in the mindset and other information from the external environment and the comfort zone. Meanwhile, trust evaluators serve as gatekeepers that assess the reliability of the information and give a "priority pass" to trusted information so that it can easily be accepted without going through the normal full evaluating process. In contrast, if the information is distrusted, it can be rejected quickly. As the human body needs the energy to function, the neuronal computation activities of the multi-filtering system also consume energy. The more information being processed, the more energy is required. Based on this rationale, it is plausible to say that the "priority pass" mechanism of the trust evaluator is a means to conserve energy during the multi-filtering process.

The assumption that an individual uses trust as a mechanism to conserve energy is also aligned with biological evidence. Living organisms on Earth strive to maintain a highly ordered state as the biosphere evolves in the direction of increasing complexity (Schrödinger, 1944). All biological systems are limited by their usable energy capacity, and organisms have constantly been evolving to maximize energy efficiency due to the harsh competition for resources. Various energy-saving mechanisms can be found everywhere in nature, from non-biological to all evolutional levels of biological organisms (Trenchard \& Perc, 2016). The human brain is only about $2 \%$ of body mass, but it uses about $20 \%$ of oxygen supply during resting state (Mink, Blumenschine, \& Adams, 1981). Neuronal computation requires a lot of energy, and thus the limited energy supply for the brain is a big constraint for its information processing capacity (Harris, Jolivet, \& Attwell, 2012). Following synaptic plasticity mechanisms, learning by creating many associations between values with high accuracy is very energetically expensive 
(Li \& van Rossum, 2020). The brain can utilize sparse coding to "squeeze" information together to save energy, making information processing more efficient (Haider et al., 2010).

The universality of the Internet and personal digital devices has changed the major information diffusion modes from traditional newspapers and word-of-mouth to social media and social networks. Current social platforms such as Facebook, Twitter, We-chat and forums enable individuals to access vast sources of information on an unprecedented scale and in a short period of time. Due to such an enormous amount of information being absorbed despite limited energy capacity, it is impossible for an individual to fully evaluate every piece of information that enters their mind. Thus, given that an individual needs to filter a certain amount of information to function well, the trust evaluator's "priority pass" mechanism has to be employed more frequently to improve the efficiency of information processing when the exposure to information in the environment increases.

Putting trust (or distrust) on a source of information will apply the priority effect (positive or negative, respectively) on all or most information with similar values coming from that source. For example, distrust toward the government will make people hastily discard information coming from there because the source itself is perceived as untrustworthy. On the other hand, trusting an individual such as a political figure can make people hastily accept their statements as being true. As a well-known example, despite former United States President Donald Trump's lack of sufficient medical knowledge background, many supporters believed and followed his endorsement of using hydroxychloroquine for COVID-19 treatment - which the World Health Organization strongly advises against (World Health Organization, 2021).

When a certain value has been integrated into one's mindset, it is used as a reference for evaluating other related information. Thus opposite values will be perceived with higher cost and become less likely to be accepted because of the energy constraint. The more reinforced the belief, the more difficult and energetically expensive it is to change one's mind. Therefore, it is a race to see who would be the first to provide appealing information that could enter mindset faster for every issue.

Misinformation spreaders can take advantage of the function of trust by using the strategy of "half-truth" - presenting commonly trusted information (such as obvious facts or published scientific results) before misinterpreting them and blending in personal claims (such as baseless conspiracy theories). This approach creates trust toward the speakers first to make it easier for listeners to (carelessly) accept subsequent information from them.

Although trust as an energy-saving mechanism is a potential concept to study misinformation and other psychological processes, further theoretical development and empirical validation are required. To further empirically examine the Mindsponge mechanism of trust, the Bayesian Mindsponge Framework (BMF) (Nguyen \& Le, 2021) has been shown to be advantageous and effective in past studies on religiosity (Q.-H. Vuong, Nguyen, \& Le, 2021b) and healthcare service (Q.-H. Vuong, Le, La, \& Nguyen, 2021). 


\section{References}

Center for Countering Digital Hate. (2021). The Disinformation Dozen. Retrieved from https://www.counterhate.com/disinformationdozen

Haider, B., Krause, M. R., Duque, A., Yu, Y., Touryan, J., Mazer, J. A., \& McCormick, D. A. (2010). Synaptic and Network Mechanisms of Sparse and Reliable Visual Cortical Activity during Nonclassical Receptive Field Stimulation. Neuron, 65(1), 107-121. doi:10.1016/j.neuron.2009.12.005

Harris, Julia J., Jolivet, R., \& Attwell, D. (2012). Synaptic Energy Use and Supply. Neuron, 75(5), 762-777. doi:10.1016/j.neuron.2012.08.019

Li, H. L., \& van Rossum, M. C. W. (2020). Energy efficient synaptic plasticity. eLife, 9, e50804. doi:10.7554/eLife.50804

Loomba, S., de Figueiredo, A., Piatek, S. J., de Graaf, K., \& Larson, H. J. (2021). Measuring the impact of COVID-19 vaccine misinformation on vaccination intent in the UK and USA. Nature Human Behaviour, 5, 337-348. doi:10.1038/s41562-021-01056-1

Mink, J. W., Blumenschine, R. J., \& Adams, D. B. (1981). Ratio of central nervous system to body metabolism in vertebrates: its constancy and functional basis. American Journal of Physiology-Regulatory, Integrative and Comparative Physiology, 241(3), R203-R212. doi:10.1152/ajpregu.1981.241.3.R203

Nguyen, M.-H., \& Le, T.-T. (2021). Bayesian Mindsponge Framework. In Scholarly Community Encyclopedia. Basel: MDPI AG.

Nguyen, M.-H., Le, T.-T., Nguyen, H.-K. T., Ho, M.-T., Nguyen, H. T. T., \& Vuong, Q.-H. (2021). Alice in Suicideland: Exploring the Suicidal Ideation Mechanism through the Sense of Connectedness and Help-Seeking Behaviors. International Journal of Environmental Research and Public Health, 18(7), 3681. doi:10.3390/ijerph18073681

Rapp, D. N., \& Salovich, N. A. (2018). Can't We Just Disregard Fake News? The Consequences of Exposure to Inaccurate Information. Policy Insights from the Behavioral and Brain Sciences, 5(2), 232-239. doi:10.1177/2372732218785193

Satariano, A., \& Alba, D. (2020). How a 5G Coronavirus Conspiracy Theory Fueled Arson and Harassment in Britain. The New York Times. Retrieved from https://www.nytimes.com/2020/04/10/technology/coronavirus-5g-uk.html

Schrödinger, E. (1944). What is life? : Cambridge University Press.

Trenchard, H., \& Perc, M. (2016). Energy saving mechanisms, collective behavior and the variation range hypothesis in biological systems: A review. Biosystems, 147, 40-66. doi:10.1016/j.biosystems.2016.05.010

Vuong, Q.-H., Le, T.-T., La, V.-P., \& Nguyen, M.-H. (2021). The psychological mechanism of Internet information processing for post-treatment evaluation: implications for healthcare system development. Retrieved from https://osf.io/qnywu/

Vuong, Q.-H., Nguyen, M.-H., \& Le, T.-T. (2021a). Home Scholarly Culture, Book Selection Reason, and Academic Performance: Pathways to Book Reading Interest among Secondary School Students. European Journal of Investigation in Health, Psychology and Education, 11(2), 468-495. doi:10.3390/ejihpe11020034

Vuong, Q.-H., Nguyen, M.-H., \& Le, T.-T. (2021b). A mindsponge-based investigation into the psycho-religious mechanism behind suicide attacks. Warsaw: Sciendo.

Vuong, Q. H., \& Napier, N. K. (2015). Acculturation and global mindsponge: An emerging market perspective. International Journal of Intercultural Relations, 49, 354-367. doi:10.1016/j.ijintrel.2015.06.003 
Waszak, P. M., Kasprzycka-Waszak, W., \& Kubanek, A. (2018). The spread of medical fake news in social media - The pilot quantitative study. Health Policy and Technology, 7(2), 115-118. doi:10.1016/j.hlpt.2018.03.002

World Health Organization. (2021). WHO Living guideline: Drugs to prevent COVID-19. Retrieved from https://www.who.int/publications-detail-redirect/WHO-2019-nCoVprophylaxes-2021-1 